\title{
BMJ Open Arrival by ambulance in acute heart failure: insights into the mode of presentation from Acute Studies of Nesiritide in Decompensated Heart Failure (ASCEND-HF)
}

\author{
Justin A Ezekowitz, ${ }^{1}$ Mohua Podder, ${ }^{1}$ Adrian F Hernandez, ${ }^{2}$ Paul W Armstrong, ${ }^{1}$ \\ Randall C Starling, ${ }^{3}$ Christopher M O'Connor, ${ }^{2}$ Robert M Califf ${ }^{2}$
}

To cite: Ezekowitz JA, Podder M, Hernandez AF, et al. Arrival by ambulance in acute heart failure: insights into the mode of presentation from Acute Studies of Nesiritide in Decompensated Heart Failure (ASCEND-HF). BMJ Open 2016;6:e010201. doi:10.1136/bmjopen-2015010201

- Prepublication history and additional material is available. To view please visit the journal (http://dx.doi.org/ 10.1136/bmjopen-2015010201).

Received 6 October 2015 Accepted 13 January 2016

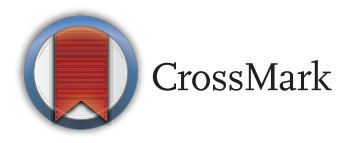

${ }^{1}$ Division of Cardiology, University of Alberta and Mazankowski Alberta Heart Institute, Edmonton, Alberta Canada

${ }^{2}$ Duke Clinical Research Institute, Durham, North Carolina, USA

${ }^{3}$ Department of

Cardiovascular Medicine, Cleveland Clinic, Cleveland, Ohio, USA

Correspondence to Dr Justin A Ezekowitz; jae2@ualberta.ca

\section{ABSTRACT}

Objectives: Limited data exist assessing the relationship between ambulance versus self-presentation and outcomes in patients with acute heart failure (AHF).

Setting: Clinical trial sites in North America. Participants: 1068 patients enrolled in the Acute Studies of Nesiritide in Decompensated Heart Failure (ASCEND-HF) trial.

Primary and secondary outcome measures: The association between ambulance use and dyspnoea improvement, 30-day mortality or HF rehospitalisation and 180-day mortality.

Results: Of the 1068 patients in the substudy, 832 $(78 \%)$ self-presented (SP) and $236(22 \%)$ patients presented via ambulance. Patients presenting via ambulance were older, more likely to be female, have a higher ejection fraction but similar natriuretic peptide levels as patients who SP. Patients presenting by ambulance (compared with SP) trended towards more dyspnoea improvement at $6(p=0.09)$ and $24 \mathrm{~h}$ $(p=0.10)$. The co-primary end point (30-day mortality or HF rehospitalisation) was similar between groups (ambulance $12.2 \%$ vs SP $11.4 \%, p=0.74$ ). Patients who presented by ambulance had a higher 30 -day and $180-$ day mortality rate than those who SP (30-day: $4.3 \%$ vs $2.2 \%, p=0.08 ; 180$-day: $15.1 \%$ vs $10.3 \%, p=0.04$ ). After adjustment for baseline characteristics, patients arriving by ambulance (compared with SP) had a 2-fold high risk of 30 -day mortality (OR $2.12,95 \% \mathrm{Cl} 0.94$ to 4.79 ), but no relationship to the composite of 30-day mortality/HF rehospitalisation (OR 1.01, 95\% $\mathrm{Cl} 0.63$ to 1.63).

Conclusions: Among patients with AHF, 30-day and 180-day mortality is 1.5-2 times higher for those with presenting via ambulance compared with patients who self-present. Understanding patient-related and systemrelated factors of ambulance use for patients with AHF is important.

Trial registration number: NCT00475852.

\section{INTRODUCTION}

Heart failure (HF) is a major cause of death and disability. This is especially evident in

\section{Strengths and limitations of this study}

- This is a prospectively designed study that collected information from a multinational clinical trial.

- Unknown potential confounders may limit interpretation.

- High quality data and patient details available.

- Generalisability may be limited as this study was done as part of a pragmatic clinical trial.

the emergency department (ED), which continues to be the primary location where patients receive care prior to hospital admission, or are discharged. Additionally, since the acute setting of the ED serves as the initial point of entry for newly diagnosed HF, patient data pertaining to this event is important to explore.

Whereas there is extensive information in acute myocardial infarction (MI) trials, registries and population health data sets regarding the use of the ambulance, ${ }^{1}{ }^{2}$ there is little describing the mode of presentation (either ambulance or self-present) in acute HF (AHF). In patients with acute MI, extensive networks and resources as well as public health initiatives have been developed to enhance and improve the use of ambulance services. Patients with acute MI using ambulance services are at higher baseline risk and higher risk for subsequent short-term (eg, 30-day) clinical events.

Data from Canadian patients enrolled in Acute Studies of Nesiritide in Decompensated Heart Failure (ASCEND-HF) showed that $27 \%$ of trial and $52 \%$ of registry patients arrived by ambulance. ${ }^{3}$ Other population health data have also shown that $50 \%$ of patients admitted to hospital for AHF 
arrive via ambulance, and although associated with other high-risk features, ambulance arrival independently predicted early mortality. ${ }^{4-6}$ However, uncertainty exists with respect to the use of emergency services across a broader group of patients including those that utilise an emergency service call as the start of their healthcare encounter. Furthermore, there has been no description of the detailed timeline of care provided from symptom onset through hospital admission of patients with AHF that would mirror information available on patients with acute MI.

Accordingly, we designed a substudy to collect prehospital data on individuals enrolled in the ASCEND-HF trial. We hypothesised that patients who presented via ambulance would be at higher risk for early mortality after controlling for patient-related factors. We further describe the relationship of mode of presentation on the clinical outcomes including mortality, rehospitalisation and dyspnoea.

\section{METHODS}

\section{Study design}

The study design and results of the ASCEND-HF trial have been previously published. ${ }^{2}{ }^{5}$ Briefly, the ASCEND-HF study evaluated nesiritide versus placebo in 7007 patients with acute decompensated HF (ADHF) enrolled within $24 \mathrm{~h}$ of first intravenous HF-related treatment. Participants were required to have each of the following at time of randomisation: dyspnoea at rest or with minimal activity; $\geq 1$ accompanying sign (respiratory rate $\geq 20$ breaths/min or pulmonary congestion/ oedema with rales $\geq 1 / 3$ base); and $\geq 1$ objective measure of $\mathrm{HF}$ (evidence of congestion/oedema on chest X-ray; B-type natriuretic peptide (BNP) $\geq 400 \mathrm{pg} /$ $\mathrm{mL}$ or $N$-terminal pro-BNP $\geq 1000 \mathrm{pg} / \mathrm{mL}$; pulmonary capillary wedge pressure $>20 \mathrm{~mm} \mathrm{Hg}$; or left ventricular ejection fraction $<40 \%$ in the previous 12 months). There was no requirement for patients to have presented via emergency services (eg, ambulance) or self-present, but all patients had to be admitted to hospital for treatment of the ADHF.

\section{Early process of care substudy design}

Patients in the prospectively planned early process of care substudy were enrolled between April 2009 and August 2010 at 206 sites in Canada and the USA. Patients from outside North America were also eligible but based on differences in the structure and organisation of emergency medical services (EMS), inconsistent data capture and enrolment into this substudy, these patients $(\mathrm{n}=1742)$ were excluded from further analysis. The patients were required to provide written informed consent for the ASCEND-HF trial.

Sites were asked to provide information on whether or not patients had called emergency services (eg, 911, $000,112)$, and if they arrived via ambulance or selfpresented (SP; eg, walk-in, come by private vehicle or public transit). Additional information was collected on time from symptom onset, hospital or ED contact, first laboratory work and time of admission. Length of stay and other times were captured in the main case report form. Symptom onset was defined as the date and time of the qualifying HF event. Hospital arrival time was defined as when the patient arrived to the ED. Time to first medication was defined as when the study drug or placebo infusion was started. Most patients ( $\mathrm{n}=960$, $89.9 \%)$ received an intravenous diuretic after presentation to the ED and before the study drug; however, the times were not recorded.

\section{Statistical analysis}

The aim of this study was to compare two groups of patients: those who presented by ambulance versus SP. Baseline characteristics for the selected cohort were tabulated according to presentation groups. Categorical variables were summarised through percentages and continuous variables by median and IQR for each presentation groups separately. Wilcoxon rank-sum test was applied to test the unadjusted differences between groups for continuous variables, and the $\chi^{2}$ test for the discrete variables.

The relationship between the mode of presentations and outcomes (both short term and long term) was explored through multivariable analysis. Multivariable logistic regression and Cox proportional hazard models were applied for short-term (30-day) and long-term (31-day to 180-day) outcomes, respectively. The model for 30-day all-cause death was adjusted for age, blood urea nitrogen (BUN), sodium, qualifying episode of dyspnoea and systolic blood pressure (SBP) from baseline. Both models for composite event of 30-day death or HF rehospitalisation and 31-day to 180-day death were adjusted based on age, BUN, creatinine clearance, sodium, qualifying episode of dyspnoea, SBP at baseline; and history of cerebrovascular disease, depression, HF, chronic respiratory disease. The adjusted list of covariates was validated through the ASCEND-HF risk model. ${ }^{7}$ Dyspnoea, measured using a Likert scale, at 6 and $24 \mathrm{~h}$ was compared using the same methods as the overall trial. ${ }^{89}$

All the statistical tests have been performed two-sided with $\mathrm{p}<0.05$ indicating statistical significance with SAS software, V.9.3 (SAS Institute, Cary, North Carolina, USA).

\section{RESULTS}

Of the 7007 patients in ASCEND-HF, 4075 were enrolled before the substudy started to collect data, of which 2081 patients were enrolled in North America (figure 1). Compared with the patients enrolled prior to the substudy in North America, there were no clinically important differences (see e-appendix, e-table 1). Among the cohort of 1068 patients in the substudy, 832 patients SP and 236 patients presented via ambulance or other emergency services. 


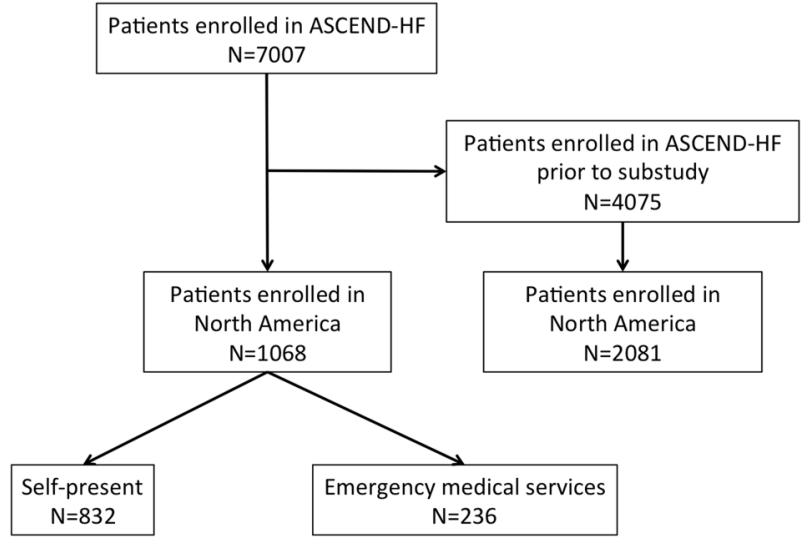

Figure 1 Cohort diagram for Acute Studies of Nesiritide in Decompensated Heart Failure (ASCEND-HF) substudy.

Patients presenting by ambulance were more likely to be female, were older, weighed less and had a higher ejection fraction than those who SP (table 1). The use of implantable cardioverter-defibrillators (ICDs), $\beta$-blockers and aldosterone antagonists was lower in patients presenting by ambulance, but ACE inhibitor or angiotensin receptor blocker use was similar to those who SP. Haemoglobin levels were lower in patients presenting via ambulance compared with those SP. Natriuretic peptide levels were numerically (but not statistically) lower in patients presenting via ambulance compared with those SP.

Patients presented to hospital a median of $26.1 \mathrm{~h}$ after symptom onset (IQR 1.4-130.3 h) and this tended to be shorter for patients who presented by ambulance (median $22.7 \mathrm{~h}$ (IQR 1.5-71.1 h)) compared with those who SP (median $28.7 \mathrm{~h}$ (IQR $1.4-152.3 \mathrm{~h} ; \mathrm{p}=0.06)$ ). Patients underwent randomisation into the ASCEND-HF trial a median of $45.2 \mathrm{~h}$ after symptom onset (IQR 21.3$148.3 \mathrm{~h})$, and this was shorter for patients presenting by ambulance (39.4 h (IQR 17.5-88.2 h)) compared with patients who SP (47.8 h (IQR 21.7-169.8 h; p=0.02)). Accordingly, patients who arrived by ambulance had a shorter time from hospital presentation to randomisation $(14.7 \mathrm{~h}$ (IQR 8.7-23.4 h)) than those who SP (18.7 h (IQR 7.5-22.7 h); p=0.87).

Patients arrived on weekdays $(88.5 \%)$ and weekends $(11.5 \%)$ and this differed according to mode of presentation (SP: $89.5 \%$ weekdays, $10.5 \%$ weekends; ambulance: $84.8 \%$ weekdays, $15.2 \%$ weekends; $\mathrm{p}=0.04)$. Additionally, more patients in the SP group arrived during daytime hours $(74.5 \%)$ compared with those arriving by ambulance $(50.9 \%, \mathrm{p} \leq 0.01)$.

\section{Clinical outcomes}

Patients in the substudy had a 30-day and 180-day mortality rate of $2.6 \%$ and $11.3 \%$, respectively, and this was similar to the overall ASCEND-HF study. Patients who presented by ambulance had a higher 30-day and 180-day mortality rate than those who SP (30-day: $4.3 \%$ vs $2.2 \%, \mathrm{p}=0.08$; 180 -day: $15.1 \%$ vs $10.3 \%, \mathrm{p}=0.04)$. The co-primary end point of the ASCEND-HF trial of 30-day mortality or $\mathrm{HF}$ rehospitalisation was similar between groups (ambulance $12.2 \%$ vs SP $11.4 \%, \mathrm{p}=0.74$ ). The index hospitalisation length of stay was similar between the ambulance and SP groups $(p=0.53)$. Patients presenting by ambulance (compared with those selfpresenting) had similar dyspnoea improvement at 6 $(\mathrm{p}=0.48)$ and $24 \mathrm{~h}(\mathrm{p}=0.20$; figure 2$)$.

\section{Multivariable analysis}

Using the ASCEND-HF model to adjust for baseline characteristics, patients arriving by ambulance had a twofold high risk of 30-day mortality (OR 2.12, 95\% CI 0.94 to 4.79 ), a trend towards increased 31 -day to 180 -day mortality (HR $1.70,95 \%$ CI 0.94 to 3.08 ) but no relationship to the composite of 30-day mortality/HF rehospitalisation (OR 1.01, 95\% CI 0.63 to 1.63 ) when compared with those patients who are SP. When additional variables were added, including time of presentation, weekday or weekend, none of these were independently significant predictors of the above outcomes.

\section{DISCUSSION}

In this prospectively designed substudy, we demonstrated that $22 \%$ of patients with AHF in the ASCEND-HF trial presented via ambulance. There are three principal novel observations emerging from our study: the patients presenting via ambulance: (1) are older, more likely to female and have a higher ejection fraction; (2) their risk by traditional markers including vital signs, natriuretic peptides and renal function do not differ from those who self-present; and (3) their early and adjusted mortality was twofold that of patients who self-present.

Prior studies have described the rate of ambulance use in unselected populations of patients with AHF seen in the ED. In two prior Canadian studies, between $38 \%$ and $52 \%$ of patients with AHF seen in the ED arrived via ambulance. ${ }^{10} 11$ In the study by Lee $e t a l,{ }^{10}$ patients arriving via ambulance were at an over twofold higher risk for 7-day mortality and factor into the risk score intended to predict poor outcomes. Since an ambulance trip by itself confers (to the best of our knowledge) no direct detrimental effect, this likely reflects the patient population more likely to utilise EMS: patients who are older, socially isolated and female.

Should the mode of arrival be incorporated into patient-level decisions or triage? It is likely that this feature is confounded by the above factors in addition to cost concerns, symptom duration and availability of other modes of transport, and thus captures unmeasured confounders and patient preferences rather than actually providing a risk factor linked to the disease state. This is supported by our analysis demonstrating no difference in traditional or advanced biomarkers such as BNP, yet a twofold difference in the risk of death at 30 days. 
Table 1 Baseline characteristics

\begin{tabular}{|c|c|c|c|c|}
\hline Variable & Total $(n=1068)$ & $S P(n=832)$ & EMS $(n=236)$ & p Value \\
\hline \multicolumn{5}{|l|}{ Demographics } \\
\hline Female (\%) & 35 & 33 & 44 & 0.003 \\
\hline Age (years) & $67(55,78)$ & $65(55,77)$ & $72(61,81)$ & $<0.001$ \\
\hline Weight (kg) & $87(73,106)$ & $89(74,109)$ & $83(72,102)$ & 0.001 \\
\hline Race (\%) & & & & 0.641 \\
\hline White & 61 & 62 & 58 & \\
\hline Black & 34 & 33 & 36 & \\
\hline Asian & 2 & 2 & 2 & \\
\hline Other & 3 & 3 & 4 & \\
\hline \multicolumn{5}{|l|}{ Past medical history (\%) } \\
\hline Hospitalised for HF in the last year & 46 & 45 & 48 & 0.397 \\
\hline Ischaemic aetiology of HF & 60 & 60 & 61 & 0.748 \\
\hline Prior myocardial infarction & 35 & 35 & 36 & 0.604 \\
\hline Diabetes mellitus & 49 & 49 & 50 & 0.744 \\
\hline Hypertension & 83 & 81 & 88 & 0.017 \\
\hline Atrial fibrillation & 42 & 43 & 38 & 0.159 \\
\hline ICD & 17 & 19 & 12 & 0.012 \\
\hline Biventricular pacemaker & 1.3 & 1.6 & 0.4 & 0.175 \\
\hline COPD & 27 & 26 & 29 & 0.403 \\
\hline Depression & 12 & 12 & 12 & 0.970 \\
\hline \multicolumn{5}{|l|}{ Physical examination } \\
\hline Systolic blood pressure $(\mathrm{mm} \mathrm{Hg})$ & $126(111,140)$ & $124(110,140)$ & $130(116,140)$ & 0.003 \\
\hline Diastolic blood pressure $(\mathrm{mm} \mathrm{Hg})$ & $74(65,84)$ & $74(65,85)$ & $72(64,84)$ & 0.477 \\
\hline Heart rate at rest (bpm) & $79(69,90)$ & $79(70,90)$ & $78(69,90)$ & 0.616 \\
\hline Respiratory rate (breaths/min) & $22(20,24)$ & $22(20,24)$ & $22(20,24)$ & 0.003 \\
\hline \multicolumn{5}{|l|}{ Investigations } \\
\hline Creatinine ( $\mu \mathrm{mol} / \mathrm{L})$ & $115(90,145)$ & $115(90,143)$ & $115(95,149)$ & 0.816 \\
\hline $\mathrm{BNP}(\mathrm{pg} / \mathrm{mL})$ & $1020(618,1822)$ & $1036(633,1869)$ & $986(577,1752)$ & 0.244 \\
\hline NT-pro-BNP & $4792(2661,9355)$ & $5043(2642,9398)$ & $3920(2955,7492)$ & 0.797 \\
\hline Haemoglobin (g/dL) & $123(110,135)$ & $124(110,136)$ & $120(108,133)$ & 0.034 \\
\hline Sodium (mmol/L) & $139(137,141)$ & $139(136,141)$ & $139(137,141)$ & 0.111 \\
\hline Ejection fraction (\%) & $28(20,40)$ & $25(20,40)$ & $33(21,50)$ & $<0.001$ \\
\hline \multicolumn{5}{|l|}{ Medications (\%) } \\
\hline ACE inhibitor or ARB* & 63 & 64 & 62 & 0.605 \\
\hline$\beta$-blocker ${ }^{*}$ & 74 & 76 & 67 & 0.009 \\
\hline Aldosterone antagonists* & 23 & 25 & 15 & 0.001 \\
\hline Nitrates (oral or topical)* & 24 & 24 & 26 & 0.542 \\
\hline Inotropes & 2 & 2 & 2 & 0.554 \\
\hline Vasodilators & 3 & 2 & 7 & $<0.001$ \\
\hline Use of study drug bolus & 55 & 51 & 70 & $<0.001$ \\
\hline
\end{tabular}

Values are median $(q 1, q 3)$ unless otherwise stated.

${ }^{*}$ Medications prior to hospitalisation.

ARB, angiotensin receptor blocker; BNP, B-type natriuretic peptide; COPD, chronic obstructive pulmonary disease; EMS, emergency medical services; HF, heart failure; ICD, implantable cardioverter-defibrillator; NT-pro-BNP, $N$-terminal pro-BNP; SP, self-presented.

Whether or not patients with AHF should be directed to call emergency services and be transported by an ambulance as those with acute stroke or MI are encouraged to do so via public service messages is uncertain. The prevalence of $\mathrm{HF}$ is high in the community, ${ }^{12}{ }^{13}$ and thus the implications to patients, payers and providers should be considered before deploying a community-wide public health message. Additionally and since RCT-proven therapies for treating AHF are non-existent, what additional benefit will the rapid arrival, diagnosis, triage and transport have on clinical outcomes? Research involving integrated health teams equipped with the appropriate knowledge, equipment (such as rapid turn-around biomarkers including BNP) and treatments need to be systematically studied. One such randomised trial is underway testing the utility of rapid access testing of BNP in the ambulance trying to shorten the time to therapy and potentially clinical outcomes (NCT01634425, http://www.clinicaltrials.gov). In the ED, use of biomarkers has aided in the diagnosis of $\mathrm{HF}^{14}$ provides improved discrimination when used in diagnostic scores ${ }^{1}$ and once integrated into clinical practice, is cost-effective. ${ }^{2} 15$

Early trials involving therapies available prehospital for acute MI and stroke have demonstrated a reduction in early mortality or morbidity. One AHF trial using 
Figure 2 Self-assessed

dyspnoea at 6 and $24 \mathrm{~h}$ according

to mode of presentation. EMS,

emergency medical services; SP, self-presented.

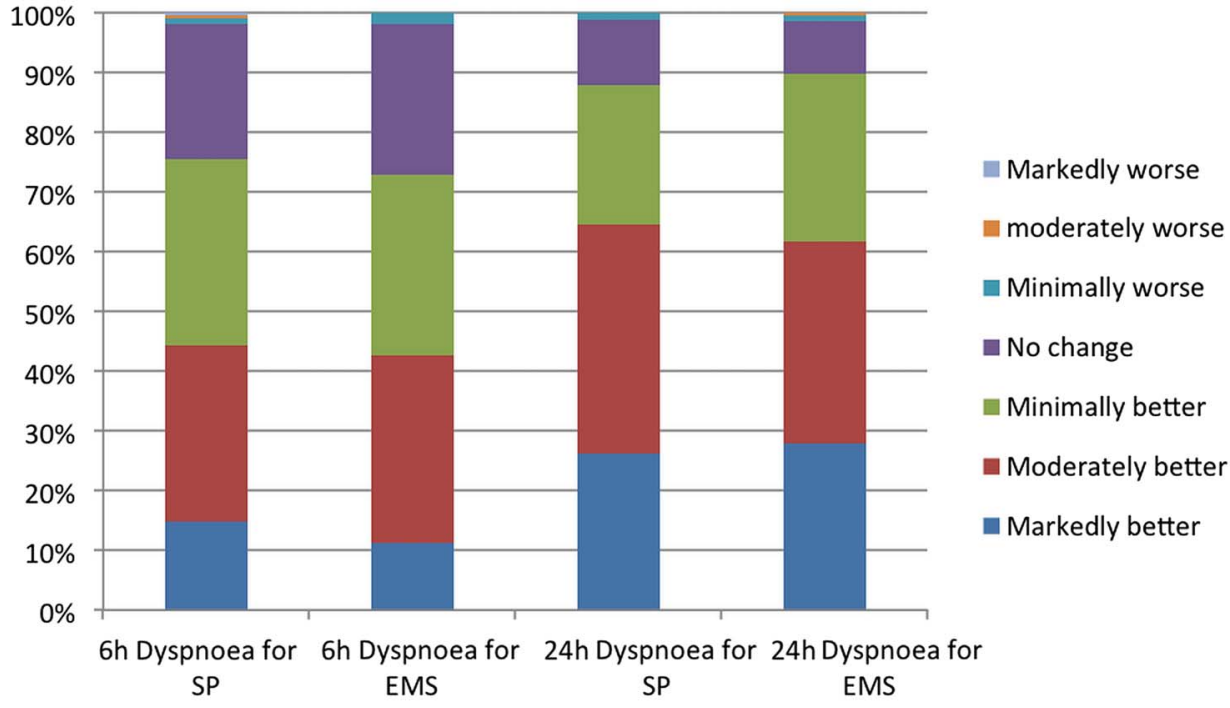

prehospital high-dose nitrates demonstrated a reduction in early adverse clinical events. ${ }^{3}{ }^{16}$ Replication of this and other novel therapies need to be tested in appropriately designed RCT, as other therapies with an early positive signal have yet to demonstrate efficacy. ${ }^{5} \quad 6 \quad 17$ Additional therapy deployed early in the ED has had mixed results for vasodilators, including nesiritide. ${ }^{9} 1012-$ 141819

We present information regarding the time of day and day of week as these have been previously linked to outcomes. ${ }^{20}$ In our study, while differences exist with more ambulance patients presenting at night and on the weekends, this may represent other social factors rather than a true difference in risk related to the patient. For example, patients who live alone may be unable to get to the ED at night, or on the weekends due to availability of family or friends to transport them to the ED and other factors such as patient preferences may play a role in mode of transportation. ${ }^{21}$ Prehospital delay has also not been linked to clinical outcomes; however, patients with $<24 \mathrm{~h}$ since symptom onset have a lower BNP and a shorter length of stay. ${ }^{22}$ Indeed, when added to the multivariable model, these factors were not independently significant predictors of clinical outcomes in ASCEND-HF.

\section{Strengths and limitations}

While this is a prospectively designed substudy of a highquality RCT, certain limitations deserve mention. First, in designing the study, we recognised the difficulty in capturing information in a global trial given the substantial differences in the meaning and delivery of ambulance services in different countries (and within countries). We therefore had to limit the data presented to that of North America where services are more homogeneous. Whether similar findings are evident elsewhere in the world is uncertain. Second, we captured detail about time from symptom onset; however, patient description of symptoms and their timing may vary based on severity of symptoms, chronicity of disease, prior similar events and other unknown confounders. The sites captured this information in an identical fashion; yet, there is limited information on the other potential confounders including the trigger for needing emergent care which may vary significantly across patient groups and regions. Third, ASCEND-HF represents a more pragmatic RCT and differences may exist in non-RCT populations due to the certainty in the diagnosis of HF; however, our findings should stimulate registry and population health studies to adequately describe and report their findings. ${ }^{11}$ ASCEND-HF, required signs, symptoms and an objective marker of HF to ensure that HF was present-prior validated criteria such as the Framingham, ${ }^{23}$ Boston $^{24}$ and other criteria need validation in the current era given the improvements in understanding the clinical phenotype of HF. Fifth, ASCEND-HF required patients to be identified and subsequently consent to participate. Both of these further limit generalisability as clinical trial sites and research team members had to be available and may have approached individuals who were less symptomatic yet still requiring hospitalisation for HF. Similarly, patients who participated may have also had less overall symptom burden or risk than patients who declined participation. Finally, we present HRs with $95 \%$ CI, and for some of our outcomes, these cross the 1.0 on the lower boundary. These should be interpreted accordingly and are considered hypothesis generating in their meaning.

\section{CONCLUSIONS}

In the ASCEND-HF trial, $22 \%$ of patients presented via ambulance and these patients were older and more likely to be female, yet did not differ in other major prognostic measures such as natriuretic peptides. However, the adjusted mortality rate was twice the rate for patients presenting via ambulance than those who self-present. Understanding patient-related and system- 
related factors of ambulance use for patients with AHF is important. Future studies should be cautious about utilising ambulance use in risk scores.

Contributors JAE, MP, AFH, PWA, RCS, CMO and RMC are solely responsible for the design and conduct of this study, all study analyses, the drafting and editing of the manuscript, and its final contents. The authors had complete control and authority over the design, data analysis, interpretation, manuscript preparation and the decision to submit this manuscript.

Funding Funding was provided by Johnson and Johnson (New Brunswick, USA) for the overall ASCEND-HF trial.

Competing interests PWA reports grants and personal fees from Boehringer Ingelheim, personal fees from Eli Lilly, grants and personal fees from Merck, personal fees from Roche and AstraZeneca, grants from GlaxoSmithKline; RMC reports grants and personal fees from Johnson and Johnson; JAE: Consulting Fees/Honoraria: Servier, Abbott Labs, Pfizer, Astra-Zeneca, Bristol-Myers Squibb; Research/Research Grants: Amgen. AFH reports grants from Janssen, BMS, GSK and Novartis. CMO reports Consulting Fees/ Honoraria: Amgen, Actelion Pharmaceuticals Ltd; Ownership/Partnership/ Principal: Biscardia, LLC; Research Grants: Otsuka, Astellas, Gilead, BG Medicine, Roche Diagnostics, Critical Diagnostics, ResMed. RCS reports Consulting Fees/Honoraria: Thoratec and HeartWare.

Patient consent Obtained.

Ethics approval The Institutional Review Board at each participating centre.

Provenance and peer review Not commissioned; externally peer reviewed.

Data sharing statement No additional data are available.

Open Access This is an Open Access article distributed in accordance with the Creative Commons Attribution Non Commercial (CC BY-NC 4.0) license, which permits others to distribute, remix, adapt, build upon this work noncommercially, and license their derivative works on different terms, provided the original work is properly cited and the use is non-commercial. See: http:// creativecommons.org/licenses/by-nc/4.0/

\section{REFERENCES}

1. Baggish A, Siebert U, Lainchbury JG, et al. A validated clinical and biochemical score for the diagnosis of acute heart failure: the ProBNP Investigation of Dyspnea in the Emergency Department (PRIDE) Acute Heart Failure Score. Am Heart J 2006;151:48-54.

2. Canto JG. Use of emergency medical services in acute myocardial infarction and subsequent quality of care: observations from The National Registry of Myocardial Infarction 2. Circulation 2002;106:3018-23.

3. Ezekowitz JA, Hernandez AF, O'Connor CM, et al. Assessment of dyspnea in acute decompensated heart failure: insights from ASCEND-HF (Acute Study of Clinical Effectiveness of Nesiritide in Decompensated Heart Failure) on the contributions of peak expiratory flow. J Am Coll Cardiol 2012;59:1441-8.

4. Takahashi M, Kohsaka S, Miyata $\mathrm{H}$, et al. Association between prehospital time interval and short-term outcome in acute heart failure patients. J Card Fail 2011;17:742-7.

5. Lee DS, Schull MJ, Alter DA, et al. Early deaths in patients with heart failure discharged from the emergency department: a population-based analysis. Circ Heart Fail 2010;3:228-35.
6. Ezekowitz JA, Bakal JA, Kaul P, et al. Acute heart failure in the emergency department: short and long-term outcomes of elderly patients with heart failure. Eur J Heart Fail 2008;10:308-14.

7. Starling RC, Hernandez AF, Armstrong PW, et al. Clinical outcomes at 180 days in the ASCEND-HF Trial: double-blind, placebo-controlled, multicenter acute study of clinical effectiveness of nesiritide in subjects with decompensated heart failure. J Card Fail 2011;17:971-1.

8. Hernandez AF, O'Connor CM, Starling RC, et al. Rationale and design of the Acute Study of Clinical Effectiveness of Nesiritide in Decompensated Heart Failure Trial (ASCEND-HF). Am Heart $J$ 2009;157:271-7.

9. O'Connor CM, Starling RC, Hernandez AF, et al. Effect of nesiritide in patients with acute decompensated heart failure. $N$ Engl J Med 2011;365:32-43.

10. Lee DS, Stitt A, Austin PC, et al. Prediction of heart failure mortality in emergent care: a cohort study. Ann Intern Med 2012;156:767-75, W-261, W-262.

11. Ezekowitz JA, Hu J, Delgado D, et al. Acute heart failure: perspectives from a randomized trial and a simultaneous registry. Circ Heart Fail 2012:5:735-41.

12. Ezekowitz JA, Kaul P, Bakal JA, et al. Trends in heart failure care: has the incident diagnosis of heart failure shifted from the hospital to the emergency department and outpatient clinics? Eur J Heart Fail 2011;13:142-7.

13. Curtis LH, Whellan DJ, Hammill BG, et al. Incidence and prevalence of heart failure in elderly persons, 1994-2003. Arch Intern Med 2008;168:418-24.

14. Maisel AS, Krishnaswamy P, Nowak RM, et al. Rapid measurement of B-type natriuretic peptide in the emergency diagnosis of heart failure. N Engl J Med 2002;347:161-7.

15. Moe GW, Howlett J, Januzzi JL, et al. N-terminal pro-B-type natriuretic peptide testing improves the management of patients with suspected acute heart failure: primary results of the Canadian prospective randomized multicenter IMPROVE-CHF study. Circulation 2007;115:3103-10.

16. Cotter G, Metzkor E, Kaluski E, et al. Randomised trial of high-dose isosorbide dinitrate plus low-dose furosemide versus high-dose furosemide plus low-dose isosorbide dinitrate in severe pulmonary oedema. Lancet 1998;351:389-93.

17. Gray A, Goodacre S, Newby DE, et al. Noninvasive ventilation in acute cardiogenic pulmonary edema. $N$ Engl J Med 2008;359:142-51.

18. Massie BM, O'Connor CM, Metra M, et al. Rolofylline, an adenosine A1-receptor antagonist, in acute heart failure. N Engl J Med 2010;363:1419-28.

19. Teerlink JR, Cotter G, Davison BA, et al. Serelaxin, recombinant human relaxin-2, for treatment of acute heart failure (RELAX-AHF): a randomised, placebo-controlled trial. Lancet 2013;381:29-39.

20. Fonarow GC, Abraham WT, Albert NM, et al. Day of admission and clinical outcomes for patients hospitalized for heart failure: findings from the organized program to initiate lifesaving treatment in hospitalized patients with heart failure (OPTIMIZE-HF). Circ Heart Fail 2008;1:50-7.

21. Sethares KA, Sosa ME, Fisher $P$, et al. Factors associated with delay in seeking care for acute decompensated heart failure. $J$ Cardiovasc Nurs 2014;29:429-38.

22. Johansson $\mathrm{P}$, van der Wal $\mathrm{M}$, van Veldhuisen $\mathrm{DJ}$, et al. Association between prehospital delay and subsequent clinical course in patients with/hospitalized for heart failure. J Card Fail 2012;18:202-7.

23. McKee PA, Castelli WP, McNamara PM, et al. The natural history of congestive heart failure: the Framingham Study. N Engl J Med 1971;285:1441-6.

24. Carlson KJ, Lee DC-S, Goroll AH, et al. An analysis of physicians' reasons for prescribing long-term digitalis therapy in outpatients. J Chronic Dis 1985;38:733-9. 